\title{
Redes de interação gênica e controle epigenético na transição saúde-doença
}

\author{
Gene interaction networks and epigenetic control in health and disease transition
}

\author{
Hátylas Azevedo ${ }^{1}$, Silvia Yumi Bando ${ }^{1}$, Fernanda Bernardi Bertonha ${ }^{1}$, \\ Carlos Alberto Moreira-Filho ${ }^{1}$
}

Azevedo H, Bando SY, Bertonha FB, Moreira-Filho CA. Redes de interação gênica e controle epigenético na transição saúde-doença / Gene interaction networks and epigenetic control in health and disease transition. Rev Med (São Paulo). 2015 out.-dez.;94(4):223-9.

\begin{abstract}
RESUMO: A utilização de técnicas de alto desempenho (highthroughput) - como microarranjos de DNA e sequenciamento de nova geração - para o estudo do genoma em doenças complexas (i.e. causadas pela interação de fatores ambientais e genéticos) tem levado à produção massiva de dados e exigido o desenvolvimento de abordagens sistemáticas para a investigação de fenômenos biológicos. Essas abordagens centram-se na integração de dados (expressão gênica global, sequenciamento de DNA, interações proteína-proteína), além da caracterização, modelagem e predição das propriedades emergentes dos sistemas biológicos, ou seja, dos processos que ocorrem em células e tecidos como resposta a mudanças ambientais e genéticas. Um exemplo dessa abordagem é o estudo de alterações transcricionais através da obtenção de redes de co-expressão gênica, ou GCNs (acrônimo para gene coexpression network) e a visualização gráfica dessas redes complexas. A análise das GCNs permite a determinação da topologia dessas redes e a avaliação de como as interações gene-gene são alteradas na transição saúde-doença. É também possível associar as propriedades topológicas dessas redes com a organização funcional do genoma. Mudanças em propriedades das GCNs, como entropia, modularidade, grau de conectividade dos nós e robustez, estão diretamente associadas à patofisiologia de determinadas doenças. As alterações em GCNs na transição saúde-doença e em resposta a fatores ambientais são mediadas por mecanismos epigenéticos - metilação do DNA, acetilação de histonas e RNAs não codificantes - que modulam a expressão dos genes. Assim, o estudo das GCNs e de seu controle por mecanismos epigenéticos têm permitido uma melhor compreensão das alterações dinâmicas envolvidas no estabelecimento e progressão das doenças complexas.
\end{abstract}

Descritores: Redes reguladoras de genes; Repressão epigenética; Genômica; Biologia de sistemas.

\begin{abstract}
The massive data generation derived from the study of complex diseases by high throughput techniques, such as DNA microarrays and new generation sequencing, has prompted the development of systematic approaches for the investigation of biological phenomena. These new approaches seek data integration, as well as the characterization, modeling and prediction of emergent properties in biological systems. Moreover, the analysis of transcriptional changes through gene coexpression networks (GCNs) has been utilized to capture the molecular mechanisms associated with complex diseases. Furthermore, the topological properties of GCNs have been associated with the functional organization of the genome. This approach has been used to identify different transition states associated with health and disease, where changes in the topological properties of GCNs, such as entropy, modularity, node centrality and network robustness are directly associated with the pathophysiological processes of specific diseases. On the other hand, epigenetic mechanisms - such as histone modifications and DNA methylation and non-coding RNAs - are the transforming forces behind GCNs alterations in response to environmental factors and in the healthdisease transition. Therefore, the interplay between the genome, epigenetic mechanisms and environment represents the foundation for the study of dynamic changes that govern the development and progression of complex diseases.
\end{abstract}

Keywords: Gene regulatory networks; Epigenetic repression; Genomics; Systems biology.

1. Departamento de Pediatria, Faculdade de Medicina da Universidade de São Paulo, São Paulo, Brasil. E-mails: hatylas@usp.br, silvia.bando@hc.fm.usp.br, bertonhafb@gmail.com, carlos.moreira@hc.fm.usp.br

Correspondência para: Carlos Alberto Moreira-Filho. FMUSP, Departamento de Pediatria. Av. Dr. Enéas de Carvalho Aguiar, 647. CEP: 05403-000. Cerqueira César, São Paulo, SP. E-mail: Carlos,Moreira@hc.fm.usp.br 


\section{INTRODUÇÃO}

A identificação dos mecanismos moleculares subjacentes às doenças complexas é fundamental para o seu diagnóstico, tratamento e prognóstico adequado. Nesse sentido, a análise de alterações genômicas a partir da interação com o ambiente representa um ponto importante no estudo desse conjunto de doenças. Nos últimos anos, com o avanço das tecnologias para obtenção de dados biológicos em larga escala, como o sequenciamento de última geração e a espectrometria de massa, uma enorme quantidade de dados ficou disponível para a análise de fenômenos biológicos. Na metagenômica, por exemplo, esses avanços proporcionaram o desenvolvimento de métodos que culminaram no aprofundamento do conhecimento sobre a diversidade e evolução de ecossistemas ${ }^{1}$. Porém, as tecnologias disponíveis têm gerado muito mais dados do que os cientistas podem analisar. Além da quantidade, tais dados são também complexos e geralmente parciais, ou seja, estão relacionados somente a determinadas camadas ou subsistemas do processo biológico que se deseja estudar. Inferir algo a respeito desses achados pode ser uma tarefa extremamente complexa. Assim, o desenvolvimento de ferramentas computacionais que auxiliem a análise dessa massiva quantidade de dados é fundamental para a compreensão sistemática de um determinado fenômeno biológico. Essa abordagem não reducionista visa à utilização de técnicas de visualização, análise e modelagem de diferentes fontes de dados experimentais (genômica, transcriptômica, proteômica, metabolômica, etc.).

Considerando que vias moleculares ou perfis transcricionais podem ser representados como redes sobrepostas que são reguladas de uma maneira organizada, a utilização de metodologias computacionais para a análise de sistemas complexos representa uma estratégia interessante de investigação. A análise de redes de coexpressão gênica, ou GCNs, especificamente, permite identificar os mecanismos moleculares associados às doenças complexas e explorar propriedades emergentes da organização dos sistemas biológicos nos contextos da saúde e da doença. Esse paradigma propõe que insights obtidos a partir da análise da topologia de GCNs (categorização dos nós por seu número de ligações, "clusterização" dos nós e identificação das comunidades de nós em uma rede, etc.), assim como informações da dinâmica das mesmas (i.e. mudanças da hierarquia dos nós e da estrutura de comunidades em resposta a fatores externos e intrínsecos ao genoma) possam ser utilizados para a identificação de biomarcadores e alvos terapêuticos associados à transição saúde-doença ${ }^{2}$. Além disso, o papel da epigenética na manutenção em longo prazo das alterações topológicas nas redes associadas a determinados fenótipos vem sendo estudado e representa um ponto interessante de intersecção entre a estrutura plástica do genoma e a termodinâmica de sistemas biológicos, que são organizados a partir das interações entre seus componentes moleculares.

\section{A biologia de sistemas e seu papel na era pós- genômica}

O desenvolvimento de técnicas de larga escala, principalmente com base em microarranjos de DNA, permitiu a mensuração simultânea dos níveis de expressão de milhares de genes, favorecendo o monitoramento da atividade transcricional celular em múltiplas condições ${ }^{3}$. Com a utilização desses métodos, novos insights foram obtidos sobre os mecanismos genômicos subjacentes a processos biológicos e sobre a interação entre genoma e ambiente, o que por fim levou a uma abordagem sistêmica para a identificação de genes relacionados a doenças específicas. Porém, em doenças complexas, a força motriz que direciona o metabolismo celular de um estado estável para outro - muitas vezes em resposta a mudanças ambientais - raramente deriva de mudanças num único gene e, ao contrário, envolve alterações na rede de interações gênicas ${ }^{4}$. Essas transições moldam o que chamamos de fenótipos complexos e podem ser correlacionadas com alterações específicas na hierarquia de nós e na topologia das redes de interação gênica, ou GCNs ${ }^{5}$.

A alta dimensionalidade dos dados e a natureza complexa das interações entre genes e proteínas apresentam desafios analíticos importantes para a identificação de causa e consequência, e da modelagem dos sistemas biológicos associados. Dessa maneira, abordagens integrativas para a análise dos dados genômicos são fundamentais para a investigação sistemática das alterações moleculares associadas a um determinado fenômeno biológico ${ }^{6}$. Nesse sentido, um grande desafio é a integração de informações moleculares de diferentes fontes (interatoma, genoma, transcriptoma, etc.) com o objetivo de analisar as redes moleculares de maneira dinâmica e não mais estática ${ }^{7}$.

A integração de distintas informações moleculares bem como a análise dos sistemas biológicos de maneira sistêmica é alvo da disciplina denominada Biologia de Sistemas. Essa área da biologia utiliza abordagens do tipo "top-down", como as ciências ômicas, para obter informações moleculares massivas, como o perfil de expressão gênica global, o conjunto de proteínas expressas por uma célula, o padrão de fosforilação dessas proteínas ou ainda o conjunto de metabólitos produzidos a partir de reações enzimáticas. O desafio de analisar uma quantidade enorme de dados, bem como de categorizar diferentes tipos de informações fez com que a Biologia de Sistemas tenha se tornado uma ciência em que a biologia atua de maneira integrada às ciências quantitativas, como a matemática e as ciências da computação. Métodos computacionais podem ser utilizados com o intuito de simular e modelar sistemas biológicos complexos, possibilitando a geração de conhecimentos que direcionam a investigação subsequente. 
Além disso, os esforços de integração de dados de diferentes fontes podem auxiliar na predição de como perturbações genéticas ou induzidas por drogas em células estão associadas a fenótipos complexos ${ }^{8}$.

\section{Visualização de redes de interação gênica e análise de suas propriedades topológicas}

A demonstração que dados transcriptômicos (dados quantitativos da transcrição dos genes) podem ser organizados em redes gênicas baseadas em valores de coexpressão permitiu que a Biologia de Sistemas aplicasse a teoria dos grafos para o desenvolvimento de redes de interação gênica ${ }^{9}$. As redes são representadas na teoria dos grafos por meio de nós conectados a arestas. Em análises transcriptômicas, os nós representam genes e as arestas representam as conexões entre esses genes, obtidas a partir de dados de co-expressão entre os genes. Essas redes de interação são construídas a partir de correlações de covariância gene-gene, utilizando medidas como os coeficientes de correlação de Pearson, ou de postos de Spearman ${ }^{10}$. Genes apresentando padrões de expressão similares são designados com uma interação entre eles, permitindo visualizar os padrões de correlação entre os genes estudados (Figura 1A).

A

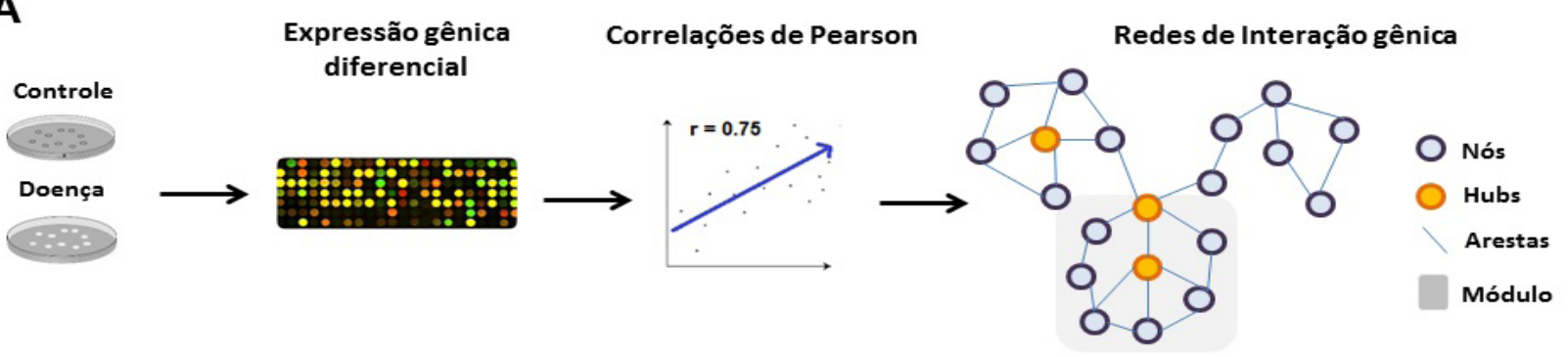

B $\Delta$
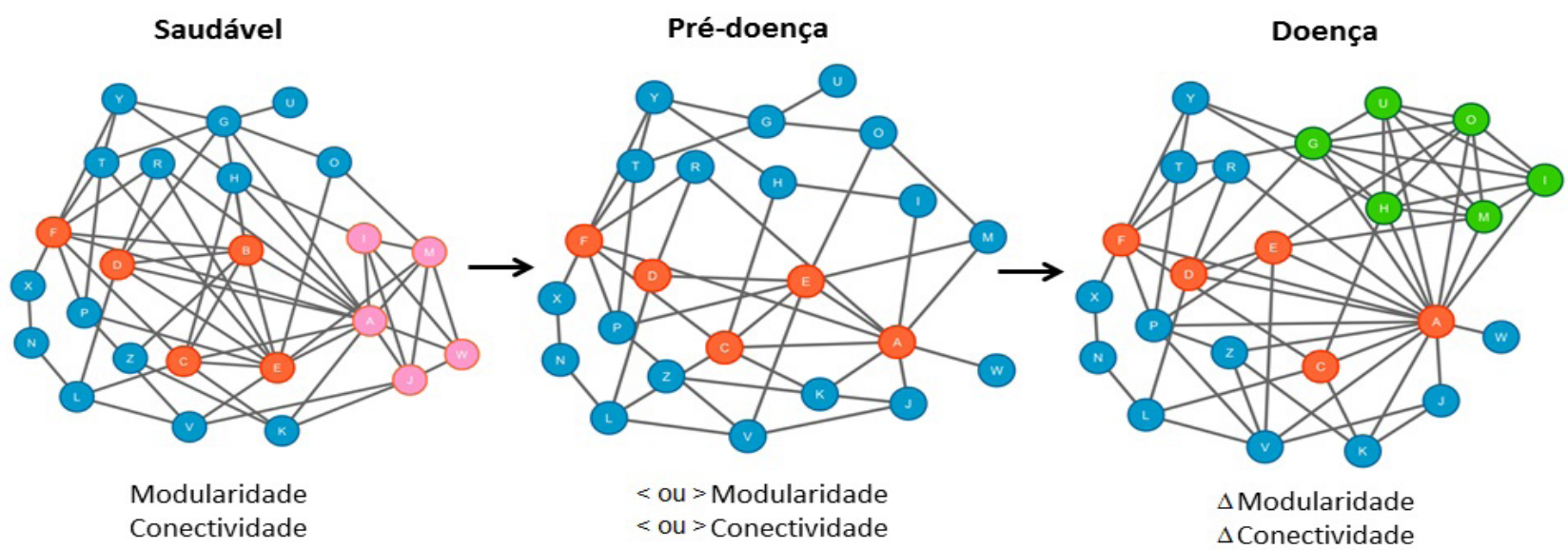

Figura 1. Pipeline para desenvolvimento de redes de interação gênica e modificações nas propriedades topológicas dessas redes durante a transição saúde-doença. A) Após a comparação entre o perfil de expressão gênica entre um grupo controle e um grupo associado a uma doença, interações baseadas em valores de co-expressão são determinadas entre os genes, utilizando medidas de correlação como a correlação de Pearson. Nessas redes, cada gene é representado por um nó e cada interação é representada por uma aresta. A partir de medidas de centralidade e de clusterização dos nós, pode-se determinar nós com maior centralidade (hubs) e módulos funcionais, representados por subgrafos em que os nós participantes apresentam alta densidade de conexões entre si. B) Análise de redes de interação gênica na transição saúde-doença. Durante a transição saúde-doença, propriedades topológicas dessas redes tais como modularidade e conectividade são alteradas, levando à formação de novos módulos funcionais bem como a um ganho de conectividade de nós específicos.

A visualização e modelagem de sistemas reais por meio do mapeamento das propriedades topológicas em redes complexas têm revelado a complexidade molecular associada a um determinado fenômeno biológico. Algumas propriedades das redes são de particular interesse, pois revelam informações sobre a hierarquia topológica dessas redes de acordo com medidas de centralidade. A organização das redes de maneira livre de escala, por exemplo, indica que essas redes não são organizadas de maneira randômica, sendo que a maior parte dos nós apresenta um número baixo de conexões enquanto alguns nós possuem um número elevado de conexões. Nós com maior centralidade, 
Azevedo H, et al. Redes de interação gênica e controle epigenético na transição saúde-doença.

denominados "hubs", apresentam maior relevância na manutenção da estrutura da rede e, consequentemente, também apresentam maior relevância biológica ${ }^{11}$. De fato, hubs estão associados com essencialidade e conservação evolutiva em redes biológicas ${ }^{12}$. A centralidade de um nó pode ser calculada de diversas maneiras, por exemplo, utilizando o número total de conexões de um nó ("node degree"), o número de caminhos mínimos que passam pelo nó ("node betweenness"), a proximidade de um nó em relação a outros nós ("closeness"), a razão entre arestas existentes e arestas possíveis entre vizinhos de um determinado nó (coeficiente de clusterização), entre outros $^{13}$.

Novos métodos têm sido desenvolvidos para a visualização e análise de redes de interação gênica em doenças complexas, incluindo a utilização de medidas de centralidade não convencionais para a caracterização hierárquica de nós ${ }^{14}$, ou ainda a determinação da estrutura modular das redes utilizando algoritmos de detecção de módulos ${ }^{15}$. De acordo com a hipótese de organização modular em redes biológicas, uma doença é representada pela perturbação local dos módulos (subgrafos) associados àquela doença. Essas perturbações podem estar associadas com a remoção de uma proteína (a partir da mutação no gene que codifica essa proteína), a ruptura de uma interação entre duas proteínas, ou ainda pela modificação na força da interação entre dois nós ${ }^{16}$.

A análise do grau de organização de uma rede utilizando medidas termodinâmicas, como a entropia, também demonstrou que células tumorais apresentam um ganho entrópico global, embora oncogenes estejam associados a uma redução na entropia da rede ${ }^{17}$. Vale notar que esse aumento na entropia global da rede está relacionado à sua topologia livre de escala (distribuição da conectividade dos nós segue uma lei de potência) e também ao aumento da expressão gênica de $h u b s^{18}$. De fato, genes categorizados como hubs em redes de interação gênica apresentam uma maior contribuição para sua entropia global e também estão associados com a letalidade (proteínas cuja ausência é letal para a célula) do sistema biológico estudado ${ }^{19}$. Em conjunto, esses dados sugerem que a elucidação de propriedades de redes biológicas pode permitir a diferenciação entre um estado celular saudável e o estado relacionado a uma doença, sendo, portanto, fundamental para a obtenção de conhecimento sobre a fisiopatologia de uma doença.

Outra propriedade interessante de redes biológicas é sua alta resiliência contra falhas aleatórias, embora essas redes sejam vulneráveis a ataques direcionados a nós centrais ${ }^{20}$. Dessa forma, a análise da hierarquia dos nós e da estrutura modular em redes biológicas pode ser realizada para a investigação da susceptibilidade dessas redes contra a remoção de nós ou módulos específicos ${ }^{21}$. Esses conceitos têm sido utilizados na identificação de novos alvos terapêuticos em oncologia, considerando que células tumorais apresentam redes biológicas robustas que são resistentes contra fármacos que atuam com mecanismos de ação restritos ${ }^{22}$. Dessa maneira, a análise das alterações na robustez e modularidade de redes de interação gênica associadas ao tumor após a remoção de nós específicos pode auxiliar na identificação de alvos terapêuticos para superar a resistência de tumores aos quimioterápicos atuai ${ }^{23}$. De fato, foi demonstrado que a maior organização modular em redes de sinalização molecular está associada a um pior prognóstico em pacientes oncológicos ${ }^{24}$.

\section{Redes de interação gênica na investigação dos mecanismos associados às doenças complexas}

A integração de dados genômicos com a análise de redes constitui uma estratégia relevante para desvendar os mecanismos moleculares de doenças complexas. A progressão de uma doença pode ser dividida em três etapas (Figura 1B): um estado normal, um estado de pré-doença (ou estado crítico), e um estado de doença. No estado normal, as redes gênicas apresentam alta resistência e robustez às perturbações externas. $\mathrm{O}$ estado de pré-doença, por sua vez, é caracterizado como sendo o limite do estado normal: a transição entre os estados de saúde e doença pode ocorrer por insulto externo incidindo sobre redes moleculares com baixa resistência e robustez, devido à sua estrutura dinâmica. Nesta fase, o sistema é sensível a insultos externos, mas ainda é reversível para o estado normal se ocorrer uma interferência adequada. Nesse estágio, apenas uma pequena mudança nos parâmetros do sistema pode ser suficiente para determinar um colapso transiente da rede (perda de conectividade e, por vezes, subsequente perda do status livre de escala) com posterior transição para o estado de doença. O estado de pré-doença representa a transição do estado normal para o estado de doença e, portanto, é causalmente relacionado a genes associados à progressão da doença. Por fim, o estado de doença representa um estágio deteriorado, porém de alta resiliência e robustez, em que o sistema atinge um novo equilíbrio e, portanto, apresenta dificuldade em retornar ao estado normal. Assim, a identificação do estado de pré-doença é fundamental para identificar os mecanismos causais que levam à consolidação do estado de doença ${ }^{25}$. De fato, alterações topológicas em redes de interação gênica ou proteína-proteína têm sido caracterizadas na transição entre saúde e doença, ou seja, a variabilidade na centralidade de determinados nós ou ainda na organização dos módulos funcionais dessas redes estão associados à baixa estabilidade das redes no estado pré-doença.

O nosso grupo no Departamento de Pediatria da FMUSP tem aplicado esses métodos no estudo da epilepsia do lobo temporal mesial (ELTM), a forma mais comum de epilepsia de adultos, na qual um terço dos pacientes é refratário às drogas antiepilépticas. Quando os pacientes com ELTM refratária são submetidos ao tratamento 
cirúrgico para remoção do foco epiléptico, explantes do cérebro podem ser usados para investigar os mecanismos moleculares subjacentes à epilepsia refratária. Com esta abordagem, o perfil transcriptômico de explantes cirúrgicos da região CA3 hipocampal foi correlacionado com resultados de análise de textura de ressonância magnética de alta resolução. Foi descoberto que os pacientes com ELTM cujo insulto precipitante estava relacionado a convulsões febris prolongadas apresentavam um perfil molecular específico na região CA3, sugerindo que as formas febril e afebril da ELTM constituem fenótipos distintos $^{26}$. Ademais, a visualização de redes complexas combinada com o uso de medidas concêntricas revelou mecanismos patogênicos e compensatórios associados à ELTM, revelando a plasticidade do genoma e das redes de interação gênica associadas nas transições entre saúde e doença $\mathrm{a}^{27}$.

Outro trabalho de nosso grupo investigou, utilizando a análise de redes de interação gênica, os mecanismos moleculares associados ao pior prognóstico de gliomas que expressam os receptores da angiotensina $\mathrm{II}^{28}$. Foi demonstrado que as redes associadas à transcrição gênica induzida pela ativação dos receptores da angiotensina II apresentam hubs que exercem funções vitais em processos de proliferação, migração e invasão celular. Esses dados sugerem, portanto, que os efeitos pró-tumorais de fatores específicos podem ser avaliados por meio da análise de redes moduladas pelo efeito transcricional dessas substâncias em células tumorais. Além disso, a integração de dados de expressão gênica com dados de interação proteína-proteína ou ainda com dados de regulação pós-transcricional por microRNAs tem auxiliado na identificação de alvos terapêuticos associados a efeitos pró-tumorais ${ }^{29}$.

\section{Mecanismos epigenéticos de regulação gênica e sua relação com as doenças crônicas}

A epigenética é definida como o conjunto de alterações funcionais do genoma que não envolve mudanças na sequência do DNA. Os mecanismos epigenéticos atuam no genoma a partir da mudança da acessibilidade da cromatina, promovendo a regulação transcricional de muitos genes ao mesmo tempo. São exemplos de eventos epigenéticos a metilação de sequências promotoras específicas no DNA em regiões ricas em citosina e guanina, e as modificações químicas em histonas (processos de metilação e acetilação), as quais remodelam a estrutura da cromatina. Estes mecanismos são fundamentais no processo normal de desenvolvimento do organismo, porém alterações epigenéticas são também fundamentais nas transições de fase entre saúde e doença. Interessantemente, foi demonstrado que alterações epigenéticas em genes associados ao envelhecimento também apresentam correlação com o grau de conectividade de um determinado gene na rede de interação gênica associada ao envelhecimento ${ }^{30}$. Além disso, mecanismos epigenéticos estão no centro das alterações dinâmicas associadas à mudança de fenótipos celulares ${ }^{31}$, como durante o desenvolvimento da resistência a quimioterápicos em oncologia ${ }^{32}$, na progressão de determinados tipos de tumores como o câncer de mama ${ }^{33}$, ou ainda na transição epitélio-mesenquimal que ocorre durante a metástase de células tumorais ${ }^{34}$.

As origens epigenéticas das doenças são um tema de fronteira na investigação biomédica, considerando que fatores ambientais físicos, químicos ou até mesmo nutricionais podem interferir nas marcas epigenéticas no genoma e consequentemente alterar o perfil transcricional de um determinado tipo celular ${ }^{35}$. Aparentemente, componentes nutricionais podem interferir em fenômenos epigenéticos pela inibição direta de enzimas que catalisam a metilação do DNA, ou ainda pela alteração da disponibilidade de um substrato relevante para uma reação enzimática específica ${ }^{36}$. De fato, condições maternas adversas durante a gravidez promovem alterações epigenéticas no genoma do feto e consequentemente tornam as redes de interação gênica em subtipos celulares específicos pré-condicionadas a um estado patológico. Esse mecanismo é subjacente à maior predisposição dessas crianças em desenvolver doenças metabólicas e cardiovasculares na vida adulta ${ }^{37}$, embora linhas de investigação adicionais ainda sejam necessárias para a identificação do valor translacional do epigenoma no diagnóstico e prevenção de doenças crônicas. Essa linha de pesquisa, conhecida como origens desenvolvimentistas da saúde e da doença, ou $\mathrm{DOHaD}$, tem adquirido relevância crescente não apenas em Pediatria como também em muitos outros campos da Medicina ${ }^{38}$.

\section{CONCLUSÕES}

O esclarecimento dos mecanismos subjacentes às doenças complexas e a identificação de novos alvos moleculares é fundamental para seu diagnóstico, prognóstico e tratamento. Muitas linhas de evidência têm reforçado o conceito de plasticidade do genoma, que por sua vez responde, via mecanismos epigenéticos, aos agentes ambientais, sejam eles um patógeno, uma droga ou uma injúria. Considerando a complexidade de alterações moleculares após alteração do genoma por mecanismos epigenéticos, a integração dessas alterações e sua visualização em redes biológicas são fundamentais para a obtenção de uma melhor compreensão sobre as propriedades topológicas das redes que estejam diretamente relacionadas com a transição saúde-doença. Espera-se com essa abordagem: i) revelar mecanismos moleculares associados a doenças complexas; ii) identificar padrões de evolução e transição de fase na origem e progressão das doenças complexas e iii) revelar novos alvos terapêuticos para o tratamento de doenças complexas. 


\section{REFERÊNCIAS}

1. Thomas T, Gilbert J, Meyer F. Metagenomics - a guide from sampling to data analysis. Microb Inform Exp. 2012;2:3. doi: 10.1186/2042-5783-2-3.

2. Chan SY, Loscalzo J. The emerging paradigm of network medicine in the study of human disease. Circ Res. 2012;111:359-74. doi: 10.1161/CIRCRESAHA.111.258541.

3. Ideker T, Krogan NJ. Differential network biology. Mol Syst Biol. 2012;8:565. doi: 10.1038/msb.2011.99.

4. Sieberts SK, Schadt EE. Moving toward a system genetics view of disease. Mamm Genome. 2007;18:389-401. doi: 10.1007/s00335-007-9040-6.

5. Benson M, Breitling R. Network theory to understand microarray studies of complex diseases. Curr Mol Med. 2006;6:695-701. doi: 10.2174/156652406778195044.

6. Braun R. Systems analysis of high-throughput data. Adv Exp Med Biol. 2014;844:153-87. doi: 10.1007/978-1-4939-20952 28.

7. Chautard E, Thierry-Mieg N, Ricard-Blum S. Interaction networks: from protein functions to drug discovery. A review. Pathol Biol (Paris). 2009;57:324-33. doi: 10.1016/j. patbio.2008.10.004.

8. Ma'ayan A, Rouillard AD, Clark NR, Wang Z, Duan Q, Kou Y. Lean Big Data integration in systems biology and systems pharmacology. Trends Pharmacol Sci. 2014;35:450-60. doi: 10.1016/j.tips.2014.07.001.

9. Geschwind DH, Konopka G. Neuroscience in the era of functional genomics and systems biology. Nature. 2009;461:908-15. doi: 10.1038/nature08537.

10. Moreira-Filho CA, Bando SY, Bertonha FB, Silva FN, Costa LF. Methods for gene coexpression network visualization and analysis. In: Passos GA. Transcriptomics in health and disease. Switzerland: Springer International Publishing; 2014.

11. Grant, SG. Systems biology in neuroscience: bridging genes to cognition. Curr Opin Neurobiol. 2003;13:577-82. doi:10.1016/j.conb.2003.09.016.

12. Bertolazzi P, Bock ME, Guerra C. On the functional and structural characterization of hubs in protein-protein interaction networks. Biotechnol Adv. 2013;31:274-86. doi: 10.1016/j.biotechadv.2012.12.002.

13. Barabási AL, Oltvai ZN. Network biology: understanding the cell's functional organization. Nat Rev Genet. 2004;5:101-13. doi: $10.1038 / \operatorname{nrg} 1272$.

14. Costa LF, Silva FN. Hierarchical characterization of complex networks. J Statist Phys. 2006;4:841-72. doi: 10.1007/s10955006-9130-y.

15. Moreira-Filho CA, Bando SY, Bertonha FB, Iamashita P, Silva FN, Costa LF, et al. Community structure analysis of transcriptional networks reveals distinct molecular pathways for early- and late-onset temporal lobe epilepsy with childhood febrile seizures. PLoS One. 2015;10:e0128174. doi: 10.1371/journal.pone.0128174.
16. Menche J, Sharma A, Kitsak M, Ghiassian SD, Vidal M, Loscalzo J, Barabási AL. Disease networks. Uncovering disease-disease relationships through the incomplete interactome. Science. 2015;347(6224):1257601. doi: $10.1126 /$ science. 1257601 .

17. West J, Bianconi G, Severini S, TeschendorffAE. Differential network entropy reveals cancer system hallmarks. Sci Rep. 2012;2:802. doi: 10.1038/srep00802.

18. Teschendorff AE, Banerji CR, Severini S, Kuehn R, Sollich $P$. Increased signaling entropy in cancer requires the scalefree property of protein interaction networks. Sci Rep. 2015;5:9646. doi: 10.1038/srep09646.

19. Manke T, Demetrius L, Vingron M. Lethality and entropy of protein interaction networks. Genome Inform. 2005;16:15963.

20. Albert R, Jeong H, Barabasi AL. Error and attack tolerance of complex networks. Nature. 2000;406:378-82. doi:10.1038/35019019.

21. Gursoy A, Keskin O, Nussinov R. Topological properties of protein interaction networks from a structural perspective. Biochem Soc Trans. 2008;36:1398-403. doi: 10.1042/ BST0361398.

22. Azmi AS, Mohammad RM. Rectifying cancer drug discovery through network pharmacology. Future Med Chem. 2014;6:529-39. doi: 10.4155/fmc.14.6.

23. Jia J, Zhu F, Ma X, Cao Z, Li Y, Chen YZ. Mechanisms of drug combinations: interaction and network perspectives. Nat Rev Drug Discov. 2009;8:111-28. doi: 10.1038/nrd2683.

24. Takemoto K, Kihara K. Modular organization of cancer signaling networks is associated with patient survivability. Biosystems. 2013;113:149-54. doi: 10.1016/j. biosystems.2013.06.003.

25. Liu R, Li M, Liu ZP, Wu J, Chen L, Aihara K. Identifying critical transitions and their leading biomolecular networks in complex diseases. Sci Rep. 2012;2:813. doi: 10.1038/ srep00813.

26. Bando SY, Alegro MC, Amaro E Jr, Silva AV, Castro LH, Wen HT, et al. Hippocampal CA3 transcriptome signature correlates with initial precipitating injury in refractory mesial temporal lobe epilepsy. PLoS One. 2011;6:e26268. doi: 10.1371/journal.pone.0026268.

27. Bando SY, Silva FN, Costa Lda F, Silva AV, PimentelSilva LR, Castro LH, et al. Complex network analysis of CA3 transcriptome reveals pathogenic and compensatory pathways in refractory temporal lobe epilepsy. PLoS One. 2013;8:e79913. doi: 10.1371/journal.pone.0079913.

28. Azevedo H, Fujita A, Bando SY, Iamashita P, Moreira-Filho CA. Transcriptional network analysis reveals that AT1 and AT2 angiotensin II receptors are both involved in the regulation of genes essential for glioma progression. PLoS One. 2014;9:e110934. doi: 10.1371/journal.pone.0110934.

29. Huang Y, Chen HC, Chiang CW, Yeh CT, Chen SJ, Chou CK. Identification of a two-layer regulatory network of proliferation-related microRNAs in hepatoma cells. Nucleic 
Acids Res. 2012;40:10478-93. doi: 10.1093/nar/gks789.

30. West J, Widschwendter M, Teschendorff AE. Distinctive topology of age-associated epigenetic drift in the human interactome. Proc Natl Acad Sci USA. 2013;110:14138-43. doi: 10.1073/pnas. 1307242110

31. Wang P, Song C, Zhang H, Wu Z, Tian XJ, Xing J. Epigenetic state network approach for describing cell phenotypic transitions. Interface Focus. 2014;4:20130068. doi: 10.1098/ rsfs. 2013.0068

32. Housman G, Byler S, Heerboth S, Lapinska K, Longacre M, Snyder N, Sarkar S. Drug resistance in cancer: an overview. Cancers (Basel). 2014;6:1769-92. doi: 10.3390/ cancers6031769.

33. Lim YY, Wright JA, Attema JL, Gregory PA, Bert AG, Smith E, et al. Epigenetic modulation of the miR-200 family is associated with transition to a breast cancer stem-cell-like state. J Cell Sci. 2013;126:2256-66. doi: 10.1242/jcs.122275.

34. Tanaka H, Ogishima S. Network biology approach to epithelial-mesenchymal transition in cancer metastasis: three stage theory. J Mol Cell Biol. 2015;7:253-66. doi: 10.1093/ jmcb/mjv035.

35. Dolinoy DC, Jirtle RL. Environmental epigenomics in human health and disease. Environ Mol Mutagen. 2008;49:4-8. doi: 10.1002/em.20366.

36. Choi SW, Friso S. Epigenetics: A New Bridge between Nutrition and Health. Adv Nutr. 2010;1:8-16. doi: 10.3945/ an. 110.1004

37. Laker RC, Wlodek ME, Connelly JJ, Yan Z. Epigenetic origins of metabolic disease: The impact of the maternal condition to the offspring epigenome and later health consequences . Food Sci Hum Wellness. 2013;1:1-11. doi:10.1016/j.fshw.2013.03.002.

38. Portha B, Fournier A, Kioon MD, Mezger V, Movassat J. Early environmental factors, alteration of epigenetic marks and metabolic disease susceptibility. Biochimie. 2014;97:115. doi: 10.1016/j.biochi.2013.10.003. 